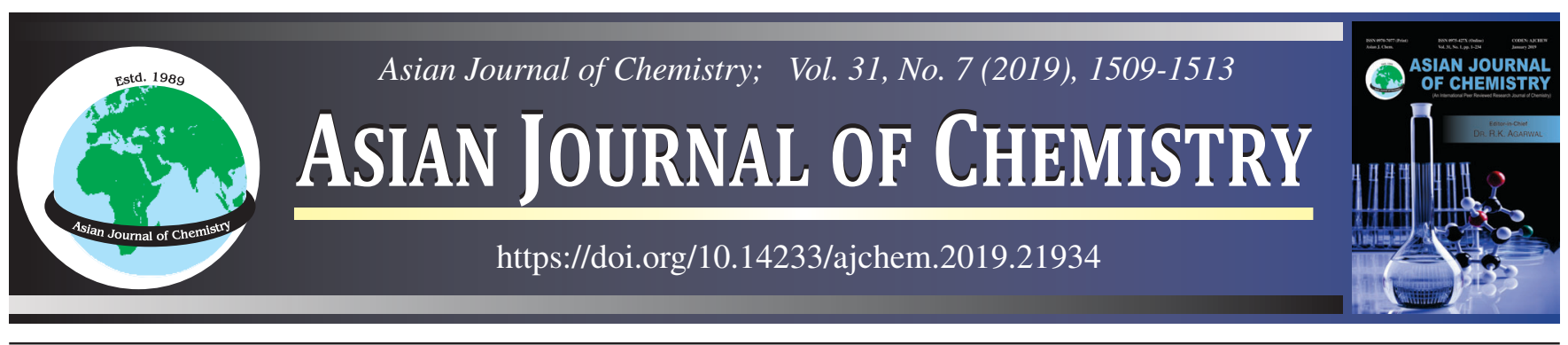

\title{
Biological Evaluation of Dihydropyrimidinone Synthesized from \\ Vanillin, Ethylacetoacetate and Urea using Gooseberry Extract
}

\section{K. Saraniya, P. Vinitha, T.R. Scotlin Blessy and Sheeba Daniel*}

Department of Chemistry and Research Centre, Holy Cross College (Autonomous), Nagercoil-629004, India

*Corresponding author: E-mail: sheebadaniel@holycrossngl.edu.in

Received: 13 February 2019;

Accepted: 11 March 2019;

Published online: 21 May 2019;

AJC-19405

The present study focuses on the synthesis, characterization and biological evaluation of dihydropyrimidinone derivative ethyl-4-(4hydroxy-3-methoxyphenyl)-6-methyl-2-oxo-1,2,3,4-tetrahydropyrimidine-5-carboxylate from vanillin, ethylacetoacetate and urea using gooseberry extract. The synthesised compound is characterized by UV-visible and FT-IR spectroscopy. The synthesised compound shows antibacterial, antifungal, antioxidant and antihelminthic activities. This natural acid catalyzed synthesis is safe, eco-friendly and does not employ any toxic materials and quantifying it as a green approach for the synthesis of organic compounds.

Keywords: Green synthesis, Dihydropyrimidinone derivative, Antimicrobial activities.

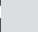

\section{INTRODUCTION}

Green chemistry approach is an eco-friendly approach which is used to prevent, or at least reduce pollution and has tremendous application in synthetic organic chemistry. This technique consists of an alternative reaction media to replace hazardous and expensive solvents consistently used in organic synthesis [1]. Green synthesis of organic compounds using solvent-free condition has gained popularity in recent years, since the majority of solvents are either toxic or flammable. Compared with conventional method the solvent free method is more convenient, simple to run, provide higher yield and shows maximum efficiency [2]. Recently fruit juice is known to be the potential organic solvent for the synthesis of pharmaceutically important organic compounds [3]. Fruit juices are used for various organic transformation reactions [4,5] and its widespread applications are due to their inexpensive, non-toxic, safe and environmentally benign nature [6].

Dihydropyrimidinones, the products of Biginelli reaction, are widely used in the pharmaceutical industries. Research has stimulated the invention of a wide range of synthetic methods for their preparation and chemical transformations. Dihydropyrimidinones and their derivatives show wide range of applications in natural, synthetic, pharmacological, therapeutic and bioorganic chemistry mainly due to their biological activities $[7,8]$. Moreover, dihydropyrimidinthiones have been suggested to be useful building blocks for synthesis of natural products, such as the batzelladine family of polycyclic marine alkaloids [9] of which batzelladine alkaloids have been found to be potent HIV gp-120-CD4 inhibitors [10].

The simple one-pot three component procedure for the synthesis of dihydropyrimidinone was first reported by Biginelli and Gazz in 1893 [11]. However, this reaction requires harsh condition, long reaction time and affords low yields, particularly when substituted aromatic aldehydes are employed. To overcome these disadvantages, several protocols have been developed to modify this reaction by means of microwave irradiation, ultrasound irradiation using various reagents. Researchers reported the synthesis of dihydropyrimidinones using various reagents or catalyst such as ionic liquids, metal oxide nanoparticles, enzyme, phase transfer catalysts, nanomagneticsupported sulphonic acid, iron(III) tosylate, bis[(L)prolinatoN,O] Zn-water, 1-glycol-3-methylimidazolium chloride, $\mathrm{Cu}$ (II) complex, $\mathrm{SiO}_{2}-\mathrm{CuCl}_{2}$, metal oxide-MWCNTs, $\mathrm{FeCl}_{3}$ and boehmite nanoparticle, nanosilica-supported tin(II) chloride, graphite, trypsin, silica sulphuric acid, etc. [12-19]. However, inspite of their potential utility, many of these reported protocols have some drawbacks such as the use of expensive reagents,

This is an open access journal, and articles are distributed under the terms of the Creative Commons Attribution-NonCommercial-ShareAlike 4.0 (CC BY-NC-SA 4.0) International License which allows readers to freely read, download, copy, distribute, print, search, or link to the full texts of its articles and to use them for any other lawful non-commercial purpose as long as the original source is duly acknowledged. 
strong acidic condition and longer reaction times. Therefore, to avoid these limitations, more efficient method is employed for the synthesis of dihydropyrimidinones.

Based on the literature survey, the present work focuses on the green synthesis of dihydropyrimidinone derivative [ethyl4-(4-hydroxy-3-methoxyphenyl)-6-methyl-2-oxo-1,2,3,4tetrahydropyrimidine-5-carboxylate] synthesized from vanillin, ethylacetoacetate and urea using gooseberry extract. Gooseberry is commonly known as amla and it belongs to the family Phyllanthaceae, one of the important herbal drugs used in Ayurvedic system of medical preparations against liver injury, atherosclerosis and diabetes [20,21]. Gooseberry is highly nutritious and is one of the richest sources of vitamin $\mathrm{C}$, amino acids and minerals. It contains several chemical constituents like tannins, alkaloids and phenols. Among all hydrolyzable tannins, gallic acid, Emblicanin A and B, ellagic acid are reported to possess biological activity. The dihydropyrimidinone derivative synthesized from gooseberry extract is characterized by UV-visible and FT-IR spectral techniques. The biological activity of the synthesized dihydropyrimidinone derivative is tested and it shows antibacterial, antifungal, antioxidant and anti-helminthic activities.

\section{EXPERIMENTAL}

Fresh and ripened gooseberries were obtained from the local market of Nagercoil, India. Vanillin, ethyl acetoacetate and urea were procured from Merck. Analytical grade ethanol was used for the recrystallisation of the products. Doubledistilled deionized water was used for the washing of precipitate.

Preparation of gooseberry extract: Ripened gooseberry was used for the preparation of the extract. This ripened fruit $(25 \mathrm{~g})$ was thoroughly washed with distilled water and cut into small pieces. Grind the pieces by a pestle and mortar and the resulting extract was filtered using Whatmann filter paper. The filtrate was collected and then centrifuged for about $8000 \mathrm{rpm}$ for about $10 \mathrm{~min}$. The supernatant extract was collected and used for the synthesis of ethyl-4-(4-hydroxy-3-methoxyphenyl)-6-methyl-2-oxo-1,2,3,4-tetrahydropyrimidine-5carboxylate from vanillin, ethylacetoacetate and urea. This gooseberry extract acts as natural acid catalyst for the synthesis of dihydropyrimidinone derivative.

Synthesis of dihydropyrimidinone derivative: The equimolar quantities of vanillin $(0.1 \mathrm{~mol})$, ethyl acetoacetate $(0.1 \mathrm{~mol})$ and urea $(0.1 \mathrm{~mol})$ were mixed in a round bottom flask. Added $1 \mathrm{~mL}$ of gooseberry extract to the mixture and refluxed at $80^{\circ} \mathrm{C}$ for $30 \mathrm{~min}$. The mixture became solid at the end of reaction and the crude product was washed with water in order to remove any water soluble impurities. The resulting yellow solid was filtered and recrystallized with ethanol to get fine crystals of ethyl-4-(4-hydroxy-3-methoxyphenyl)-6-methyl- 2oxo-1,2,3,4-tetrahydropyrimidine-5-carboxylate (Scheme-I).

The absorption spectral measurement of the synthesized compound was carried out using Shimadzu UV-1800 spectrophotometer. FTIR analysis was carried out through potassium bromide (KBr) pellet (FTIR grade) method in 1:100 ratio and spectrum was recorded using Shimadzu IR Affinity-1.

Biological evaluation: Dihydropyrimidinone derivative [ethyl-4-(4-hydroxy-3-methoxyphenyl)-6-methyl-2-oxo1,2,3,4-tetrahydropyrimidine-5-carboxylate] was synthesized from vanillin, ethyl acetoacetate and urea was subjected to investigate antimicrobial, antioxidant and antihelminthic activities.

The antibacterial activity of ethyl-4-(4-hydroxy-3-methoxyphenyl)-6-methyl-2-oxo-1,2,3,4-tetrahydropyrimidine-5carboxylate was carried out on Escherichia coli, Staphylococcus aureus, Pseudomonas fluorescens and Klebsiella pneumonia. Agar-well diffusion method was used to evaluate the antibacterial activity of the compound. Same amount $(15-20 \mathrm{~mL})$ of Mueller-Hinton agar was poured on glass petri-plates of same size and allowed to solidify. Standardized inoculum of the test organism was uniformly spread on the surface of these plates using sterile cotton swab. Four wells with a diameter of $8 \mathrm{~mm}$ (20 $\mathrm{mm}$ apart from one another) were punched aseptically with a sterile cork borer in each plate. The sample was added in each well. Then, the agar plates were incubated under suitable conditions depending upon the test microorganism. After incubation, clear zone was observed. Inhibition of the bacterial growth was measured in mm using vernier caliper.

Antifungal activity of ethyl-4-(4-hydroxy-3-methoxyphenyl)-6-methyl-2-oxo-1,2,3,4-tetrahydropyrimidine-5carboxylate was carried out on Candida albicans and Aspergillus niger. Well diffusion assay method was used to detect antifungal activities of the sample. A sterile swab was used to evenly distribute fungal culture over the potato dextrose agar medium and the plates were allowed to dry for $15 \mathrm{~min}$. Wells were then created and a pipette was used to place the sample into each well. The plates were incubated at room temperature for 3 days after which they were examined for inhibition zones. A vernier caliper was used to measure the inhibition zones.

Antioxidant activity of ethyl-4-(4-hydroxy-3-methoxyphenyl)-6-methyl-2-oxo-1,2,3,4-tetra-hydropyrimidine-5carboxylate was carried out by 2,2-diphenyl-1-picrylhydrazyl (DPPH) assay method. Ascorbic acid was used as a reference standard and dissolved in distilled water to make the stock

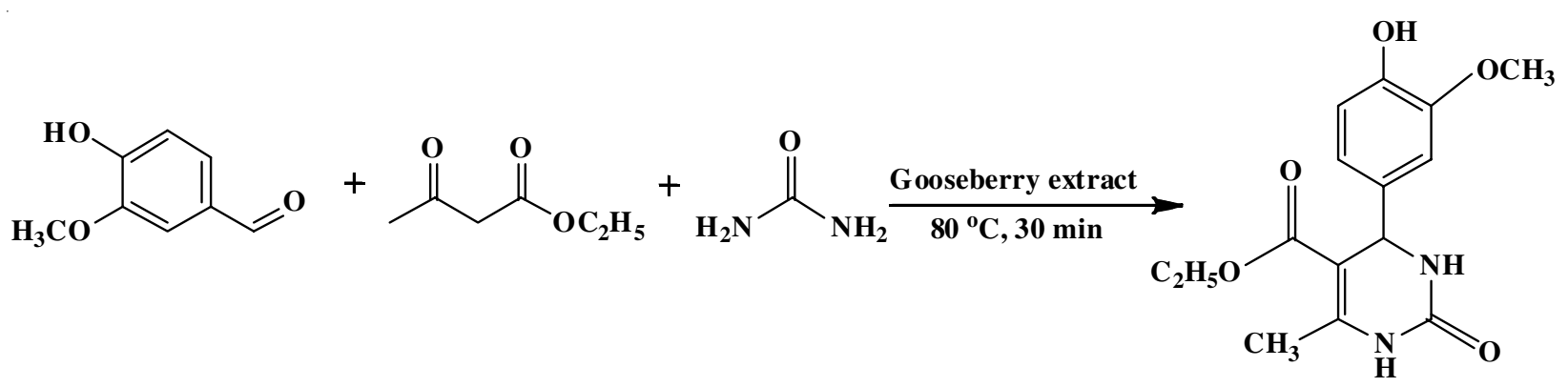

Scheme-I: Synthesis of ethyl-4-(4-hydroxy-3-methoxyphenyl)-6-methyl-2-oxo-1,2,3,4-tetrahydropyrimidine-5-carboxylate 
solution with the concentration $(1 \mathrm{mg} / 1000 \mu \mathrm{L})$. The solution of DPPH in methanol $60 \mu \mathrm{M}$ was freshly prepared before UV measurements. This solution $(3.9 \mathrm{~mL})$ was mixed with 100 $\mu \mathrm{L}$ of test solution at various concentrations. The samples were kept in the dark for $15 \mathrm{~min}$ at room temperature and the decrease in absorbance was measured. The experiment was carried out in triplicate. Control of same volume was prepared without the test sample. Ascorbic acid is used as reference and $95 \%$ methanol was used as blank. The radical scavenging activity was evaluated by measuring the absorbance at $515 \mathrm{~nm}$ and the percentage of inhibition was calculated as follows:

$$
\text { Inhibition }(\%)=\frac{(\mathrm{Abs} \text { of } \mathrm{C} \text { at } 0 \mathrm{~min}-\mathrm{Abs} \text { of } \mathrm{T})}{\mathrm{Abs} \text { of } \mathrm{C} \text { at } 15 \mathrm{~min}} \times 100
$$

where $\mathrm{C}$ is the control sample and $\mathrm{T}$ is the test solution. The antioxidant activity of the synthesized dihydropyrimidinone derivative was expressed by $\mathrm{IC}_{50}$ value. The $\mathrm{IC}_{50}$ values were determined from plots of percentage inhibition versus log inhibitor concentration and were calculated by non-linear regression analysis from the mean inhibitory values.

Antihelminthic activity of ethyl-4-(4-hydroxy-3-methoxyphenyl)-6-methyl-2-oxo-1,2,3,4-tetrahydropyrimidine-5carboxylate was carried out in Pheretima posthuma due to its anatomical resemblance with the intestinal round worm parasites of human beings. They were procured from Agriculture College, Vellayani, India TVM collected and washed with normal saline to remove all fecal matter and were used for antihelmintic activity. The suspension of extract in different concentration $(10 \mathrm{mg} / \mathrm{mL})$ was prepared by using distilled water as a suspending agent and final volume was made to 20 $\mathrm{mL}$. Distilled water was used as control. A group containing two worm's approximately equal size was released into 20 $\mathrm{mL}$ of desired concentration of the test sample in different petridishes. Observation was made for the time taken for paralysis and death. Time for death of worms was recorded after ascertaining that worms neither moved when shaken vigorously. All the results were expressed as mean \pm S.D. of two worms in each group.

\section{RESULTS AND DISCUSSION}

The role of gooseberry extract in the synthesis of biologically active ethyl-4-(4-hydroxy-3-methoxyphenyl)-6-methyl2-oxo-1,2,3,4-tetrahydropyrimidine-5-carboxylate from vanillin, ethylacetoacetate and urea is reported. The synthesized compound is characterized by UV-visible and FT-IR spectral analysis.

Synthesis of ethyl-4-(4-hydroxy-3-methoxyphenyl)-6methyl-2-oxo-1,2,3,4-tetrahydropyrimidine-5-carboxylate is a natural acid catalyzed reaction of vanillin, ethyl acetoacetate and urea. Gooseberry extract contains ascorbic acid, citric acid, gallic acid and ellagic acid. The acid present in the extracts gives $\mathrm{H}^{+}$ions for protonation. The first step in the mechanism is the condensation reaction between vanillin and urea and it forms an iminium intermediate. The iminium intermediate generated acts as an electrophile for the nucleophilic addition of the ketone and the ketone carbonyl of the resulting adduct undergoes condensation with the urea $\mathrm{NH}_{2}$ to give ethyl-4-(4hydroxy-3-methoxyphenyl)-6-methyl-2-oxo-1,2,3,4-tetrahydropyrimidine-5-carboxylate. The mechanism for the formation of dihydropyrimidinone derivate is shown in Scheme-II.

Absorption spectral analysis: The formation of ethyl4-(4-hydroxy-3-methoxyphenyl)-6-methyl-2-oxo-1,2,3,4tetrahydropyrimidine-5-carboxylate is characterized by UVvisible spectrophotometric analysis. The absorption spectrum of the synthesized compound is carried out in ethanol. The dihydropyrimidinone shows absorption peaks at 209, 250, 299 and $335 \mathrm{~nm}$ due to $\pi-\pi^{*}$ and $n-\pi^{*}$ transitions (Fig. 1). The peaks<smiles>CCOC(=O)CC(=O)[In]CC(=O)NC(O)NC(N)=O</smiles><smiles>CCOC(=O)C1=C(C)NC(=O)NC1[12OH]</smiles><smiles>CCOC(=O)C(C(C)=O)C([Al])NC(N)=O</smiles>

Scheme-II: Mechanism for the synthesis of ethyl-4-(4-hydroxy-3-methoxyphenyl)-6-methyl-2-oxo-1,2,3,4-tetrahydropyrimidine-5-carboxylate 


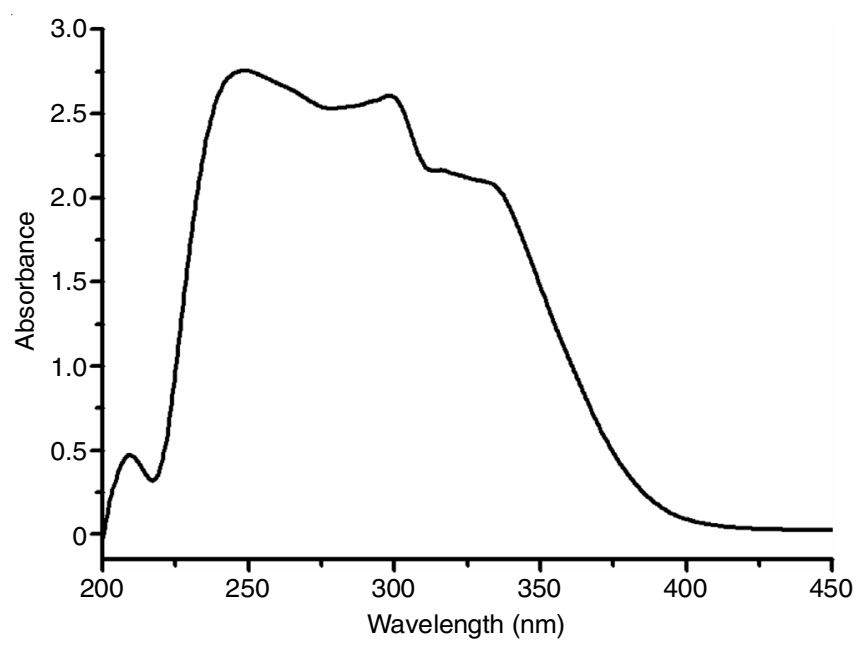

Fig. 1. Absorption spectrum of dihydropyrimidinone derivative synthesized from gooseberry extract

at 209, 299 and $335 \mathrm{~nm}$ correspond to vanillin moiety. The peak at $250 \mathrm{~nm}$ corresponds to urea and ethyl acetoacetate moiety. Thus the peaks observed in the absorption spectrum indicate the formation of dihydropyrimidinone derivative.

FT-IR spectral analysis: FT-IR spectrum of ethyl-4-(4hydroxy-3-methoxyphenyl)-6-methyl-2-oxo-1,2,3,4-tetrahydropyrimidine-5-carboxylate shows IR bands at 3395, 3050, 2923, 2850, 1672, 1589, 1509, 1380, 1300, 1164, 1010, 883, 756 and $674 \mathrm{~cm}^{-1}$, respectively (Fig. 2). The FT-IR spectrum of the synthesized dihydropyrimidinone shows a band around $3395 \mathrm{~cm}^{-1}$ indicates the $\mathrm{N}-\mathrm{H}$ stretching of amide derivative. A weak band at $3050 \mathrm{~cm}^{-1}$ is due to the stretching vibration of aromatic C-H group. The presence of weak bands at 2923 and $2850 \mathrm{~cm}^{-1}$ corresponds to the $\mathrm{C}-\mathrm{H}$ stretching of aryl and alkyl groups. The IR band at $1672 \mathrm{~cm}^{-1}$ is due to the presence of $\mathrm{C}=\mathrm{O}$ stretching of amide moiety. IR spectrum exhibits weak band at $1589 \mathrm{~cm}^{-1}$ due to the stretching vibration of aromatic $\mathrm{C}=\mathrm{C}$ bond. The band at $1509 \mathrm{~cm}^{-1}$ corresponds to the bending vibration of $\mathrm{N}-\mathrm{H}$ bond. A weak band at $1380 \mathrm{~cm}^{-1}$ is due to the $\mathrm{O}-\mathrm{H}$ bending of phenolic group. The bands at 1300 and 1164 $\mathrm{cm}^{-1}$ are due to the $\mathrm{C}-\mathrm{O}$ stretching vibrations of aromatic ester. The weak band at $1010 \mathrm{~cm}^{-1}$ is due to ring stretching. Aromatic

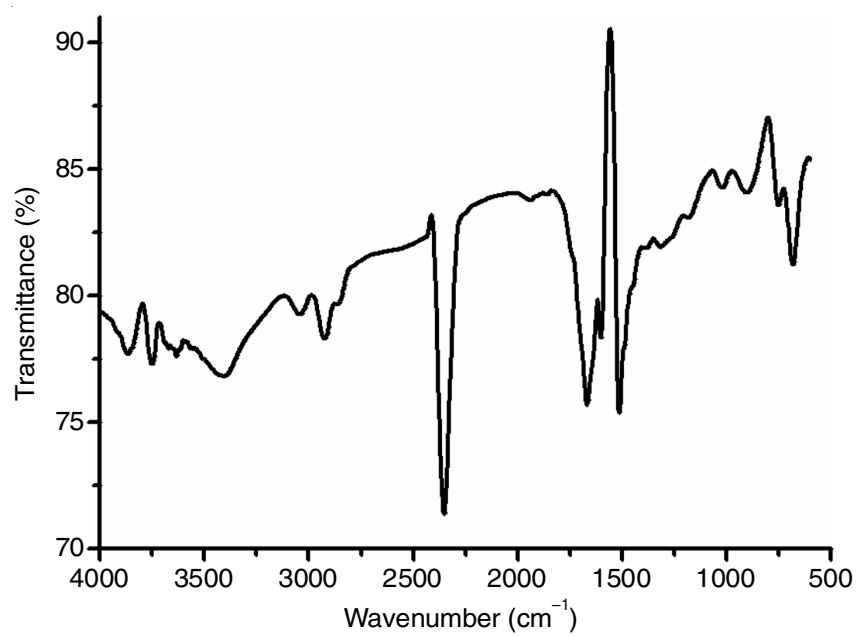

Fig. 2. FT-IR spectrum of dihydropyrimidinone derivative synthesized from gooseberry extract
C-H bending vibrations occur at 883,756 and $674 \mathrm{~cm}^{-1}$, respectively. This, FT-IR spectrum confirms the formation of dihydropyrimidinone derivative.

Antimicrobial activity: Antimicrobial activity of ethyl4-(4-hydroxy-3-methoxyphenyl)-6-methyl-2-oxo-1,2,3,4tetrahydropyrimidine-5-carboxylate was tested on Escherichia coli, Staphylococcus aureus, Pseudomonas fluorescens, Klebsiella pneumonia, Candida albicans and Aspergillus niger. The synthesized compound shows activity against the human pathogens (Table-1). Standard antibiotic disc ampicillin is used as the control for the evaluation of antibacterial activity. The inhibition zone for the control against Gram-positive and Gramnegative bacteria are in the range of $22-27 \mathrm{~mm}$. The synthesized compound ethyl-4-(4-hydroxy-3-methoxyphenyl)-6-methyl2-oxo-1,2,3,4-tetrahydropyrimidine-5-carboxylate shows higher activity against Escherichia coli than that of Staphylococcus aureus and Klebsiella pneumonia. The drug fluconazole is used as the control for the evaluation of antifungal activity. Dihydropyrimidinone derivative shows activity on Candida albicans and Aspergillus niger. The dihydropyrimidinone derivative synthesized from gooseberry extract interact directly with the microbe's outer membrane, causing the membrane to rupture and kills the microorganisms.

TABLE-1

ANTIMICROBIAL ACTIVITY OF DIHYDROPYRIMIDINONE DERIVATIVE SYNTHESIZED FROM GOOSEBERRY EXTRACT

\begin{tabular}{lc}
\multicolumn{1}{c}{ Microbes } & Zone of inhibition $(\mathrm{mm})$ \\
\hline Escherichia coli & 23 \\
Staphylococcus aureus & 12 \\
Pseudomonas fluorescens & - \\
Klebsiella pneumonia & 11 \\
Candida albicans & 12 \\
Aspergillus niger & 11 \\
\hline
\end{tabular}

Antioxidant activity: Antioxidant activity of the synthesized dihydropyrimidinone is studied by DPPH assay method. Freshly prepared DPPH solution exhibits purple colour with an absorption maximum at $515 \mathrm{~nm}$, antioxidant molecules can quench DPPH free radicals and convert them to colourless product. The synthesized compounds shows moderate antioxidant activity (Table-2). The percentage of inhibition increases with increase in the concentration of dihydropyrimidinone derivative. The $\mathrm{IC}_{50}$ value for the synthesized compound is $217.37 \mu \mathrm{g} / \mathrm{mL}$ (Fig. 3).

Antihelminthic activity: Helminthes parasite infection is a serious social and economic repercussion in developing countries. The antihelminthic assay is performed in vitro using earth worm Pheretima posthuma, since it has anatomical and

TABLE-2

ANTIOXIDANT ACTIVITY OF DIHYDROPYRIMIDINONE DERIVATIVE SYNTHESIZED FROM GOOSEBERRY EXTRACT

\begin{tabular}{ccc} 
Concentration $(\mu \mathrm{g} / \mathrm{mL})$ & OD at $515 \mathrm{~nm}$ & Inhibition $(\%)$ \\
\hline Control at $0 \mathrm{~min}$ & 0.525 & - \\
Control after $15 \mathrm{~min}$ & 0.519 & - \\
25 & 0.432 & 17.90 \\
50 & 0.396 & 24.85 \\
75 & 0.385 & 26.97 \\
100 & 0.366 & 30.60 \\
\hline
\end{tabular}




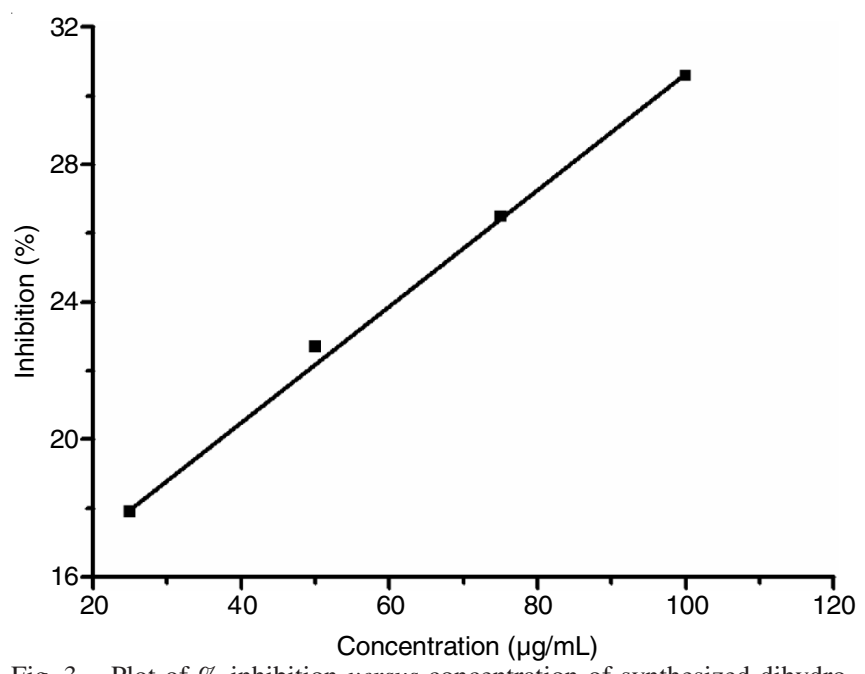

Fig. 3. Plot of $\%$ inhibition versus concentration of synthesized dihydropyrimidinone derivative

physiological similarities with the intestinal round worm of human beings for the preliminary analysis of antihelminthic activity. The results of antihelminthic activity of the synthesized compound is shown in Table-3.

\section{TABLE-3}

ANTIHELMINTHIC ACTIVITY OF DIHYDROPYRIMIDINONE DERIVATIVE SYNTHESIZED FROM GOOSEBERRY EXTRACT

\begin{tabular}{ccc}
\hline Concentration & $\begin{array}{c}\text { Paralyzed phase } \\
\text { (min taken) }\end{array}$ & $\begin{array}{c}\text { Death phase } \\
\text { (min taken) }\end{array}$ \\
\hline Control & - & - \\
Albendazole & $11.5 \pm 0.9$ & $17.9 \pm 0.6$ \\
$10 \mathrm{mg} / \mathrm{mL}$ & $7.5 \pm 1.0$ & $15.5 \pm 1.0$ \\
$5 \mathrm{mg} / \mathrm{mL}$ & $16.5 \pm 1.0$ & $38.5 \pm 1.0$ \\
\hline
\end{tabular}

The reported time taken for paralysis and death of Pheretima posthuma against albendazole at $2 \mathrm{mg} / \mathrm{mL}$ is $11.5 \pm 0.9$ and $17.9 \pm 0.6 \mathrm{~min}$ [22]. The mean paralyzing time of Pheretima posthuma against dihydropyrimidinone derivative at 10 and 5 $\mathrm{mg} / \mathrm{mL}$ is $7.5 \pm 1$ and $16.5 \pm 1 \mathrm{~min}$. The time taken for the death of Pheretima posthuma against dihydropyrimidinone derivative at 10 and $5 \mathrm{mg} / \mathrm{mL}$ is $15.5 \pm 1$ and $38.5 \pm 1 \mathrm{~min}$. The time taken for paralysis and death of Pheretima posthuma against the synthesized compound at $10 \mathrm{mg} / \mathrm{mL}$ is less than that of those in albendazole at $2 \mathrm{mg} / \mathrm{mL}$. The results revealed that synthesized dihydropyrimidinone derivative $(10 \mathrm{mg} / \mathrm{mL})$ shows higher antihelminthic activity than that of albendazole. Hence, the synthesized dihydropyrimidinone derivative is used for the treatment of helminthes in human beings.

\section{Conclusion}

An eco-friendly route for the synthesis of dihydropyrimidinone derivative from vanillin, ethylacetoacetate and urea using gooseberry extract has been investigated. The UV-visible and FT-IR spectra confirmed the formation of dihydropyrimidinone derivative. The antimicrobial activity revealed that the synthesized dihydropyrimidinone can be used for the treatment of diseases caused by microbes. The synthesized compound shows moderate antioxidant activity. Dihydropyrimidinone derivative shows antihelminthic activity and hence they can be used for the treatment of helminthes in human beings. This natural acid catalyzed synthesis is safe, does not employ any toxic materials and quantifying it as a green approach for the synthesis of organic compounds. The biological activities of the synthesized dihydropyrimidinone will trigger more interest in the synthesis of these types of compounds from the easily available starting materials.

\section{CONFLICT OF INTEREST}

The authors declare that there is no conflict of interests regarding the publication of this article.

\section{REFERENCES}

1. V. Vahabi and F. Hatamjafari, Molecules, 19, 13093 (2014); https://doi.org/10.3390/molecules190913093.

2. E. Aktoudianakis, E. Chan, A.R. Edward, I. Jarosz, V. Lee, L. Mui, S.S. Thatipamala and A.P. Dicks, J. Chem. Educ., 86, 730 (2009); https://doi.org/10.1021/ed086p730.

3. S. Patil, S.D. Jadhav and S.Y. Mane, Int. J. Org. Chem., 1, 125 (2011); https://doi.org/10.4236/ijoc.2011.13019.

4. R. Pal, Open The Org. Chem. J., 1, 47 (2013); https://doi.org/10.12966/ojoc.10.02.2013.

5. R. Pal, Indian J. Chem., 53B, 763 (2014).

6. R. Pal, S. Khannobis and T. Sarkar, Chem. J., 3, 7 (2013).

7. C.O. Kappe, Acc. Chem. Res., 33, 879 (2000); https://doi.org/10.1021/ar000048h.

8. K.S. Atwal, G.C. Rovnyak, S.D. Kimball, D.M. Floyd, S. Moreland, B.N. Swanson, J.Z. Gougoutas, J. Schwartz, K.M. Smillie and M.F. Malley, J. Med. Chem., 33, 2629 (1990); https://doi.org/10.1021/jm00171a044.

9. L. Heys, C.G. Moore and P. Murphy, Chem. Soc. Rev., 29, 57 (2000); https://doi.org/10.1039/a903712h.

10. A.D. Patil, N.V. Kumar, W.C. Kokke, M.F. Bean, A.J. Freyer, C.D. Brosse, S. Mai, A. Truneh and B. Carte, J. Org. Chem., 60, 1182 (1995); https://doi.org/10.1021/jo00110a021.

11. P. Biginelli and P. Gazz, Chim. Ital., 23, 360 (1893).

12. J.T. Starcevich, T.J. Laughlin and R.S. Mohan, Tetrahedron Lett., 54, 983 (2013); https://doi.org/10.1016/j.tetlet.2012.12.032.

13. Z.N. Siddiqui, C.R. Chim., 16, 183 (2013); https://doi.org/10.1016/j.crci.2012.10.008.

14. P. Karthikeyan, S.A. Aswar, P.N. Muskawar, P.R. Bhagat and S. Senthil Kumar, J. Organomet. Chem., 723, 154 (2013); https://doi.org/10.1016/j.jorganchem.2012.06.022.

15. G. Kour, M. Gupta, S. Paul, Rajnikant and V.K. Gupta, J. Mol. Catal. Chem., 392, 260 (2014); https://doi.org/10.1016/j.molcata.2014.05.022.

16. J. Safari and S. Gandomi-Ravandi, J. Mol. Catal. Chem., 373, 72 (2013); https://doi.org/10.1016/j.molcata.2013.02.021.

17. E. Kolvari, N. Koukabi and O. Armandpour, Tetrahedron Lett., 70, 1383 (2014); https://doi.org/10.1016/j.tet.2013.10.085.

18. K.L. Dhumaskar, S.N. Meena, S.C. Ghadi and S.G. Tilve, Bioorg. Med. Chem. Lett., 24, 2897 (2014); https://doi.org/10.1016/j.bmcl.2014.04.099.

19. Z.B. Xie, N. Wang, W.X. Wu, Z.G. Le and X.Q. Yu, J. Biotechnol., 170, 1 (2014); https://doi.org/10.1016/j.jbiotec.2013.10.031

20. S. Dasaroju and K. Gottumukkala, Int. J. Pharm. Sci. Rev. Res., 24, 150 (2014).

21. Habib-ur-Rehman, K.A. Yasin, M.A. Choudhary, N. Khaliq, Atta-urRahman, M.I. Choudhary and S. Malik, Nat. Prod. Res., 21, 775 (2007); https://doi.org/10.1080/14786410601124664.

22. A. Husain, M.M. Varshney, V. Parcha, A. Ahmad and S.A. Khan, Bangladesh J. Pharmacol., 10, 555 (2015); https://doi.org/10.3329/bjp.v10i3.23381. 